MAGNITUDES, SPECTRA, AND TEMPERATURES OF PLANETARY NUCLEI

\author{
James B. Kaler* \\ Department of Astronomy \\ University of Illinois
}

The purpose of this review is to examine the fundamental observational parameters of the central stars of planetary nebulae, namely their apparent magnitudes and gross spectral characteristics, and how these relate to the derivation or estimation of effective temperatures.

\title{
I. APPARENT MAGNITUDES
}

Probably the easiest parameter to measure for stars in general, the simple magnitude is one of the more elusive for planetary nuclei. The problem, of course, is the bright nebular background, which can in the extreme make the star impossible even to detect let alone analyze. My intention here is not to provide a listing of magnitudes - that is done in excellent fashion by Acker.et al. (1982) and their supplements - but to explore and critique the various procedures used to derive these critical numbers.

The methods in use go all the way back to eye estimates from photographic plates. Remarkably, I (Kaler 1983) needed to use one of these (from Curtis 1918) only a few years ago. Fortunately, with all the recent activity in the subject, that era has mercifully ended. Nevertheless, there is yet a vast body of photographic measurements that are still eminently usable, starting with Hubble and van Maanen, up through the extensive work by Kohoutek and Abe11: Perek and Kohoutek (1967) provide values and references. These, however, seem to suffer from a systematic trend: the nebular background apparently affects the sensitivity of the plate (in effect pre-flashes it) and makes the stars appear too bright by, crudely, 0.75 (Shaw and Kaler 1985 ). With correction, and adoption of perhaps $\pm 0^{\mathrm{m}} .4$ error (quite

* Supported by United States National Science Foundation grant AST 84 19355. 
satisfactory for much work), these magnitudes are reliable to about $\mathrm{m}=20$.

The next obvious step is to employ photoelectric methods. There are a variety of interlocking ways to approach the photoelectric problem and the elimination of the nebular background, and we will examine them to evaluate the kinds of objects for which each is most suited. The simplest approach is just to choose nebulae of such low surface brightness that the background is inconsequential. Then, like Abell (1966) in his fundamental study of large nebulae, we can simply use standard methods. His UBV values are probably correct to within a few hundredths of a division. This procedure limits us to large old nebulae, and if there is any nebular contamination will cause the magnitudes to be underestimated.

Kostjakova et al. (1968) applied UBV photometry to brighter, more compact objects. They attempted to eliminate the nebular radiation, which for these broad filters consists largely of lines, by subtracting measurements made away from the star at several positions in the nebula proper. The problem here is that planetaries are highly irregular and the surface brightness at the star may not correspond to readings made elsewhere. In addition, as they point out themselves, the nebulae must be large enough to allow such measurements. Their determinations seem to be systematically too bright by about one-half magnitude (Shaw and Kaler 1985).

Shao and Liller (1972: see Liller and Shao 1968, Liller 1978, Acker et al. 1982) improved the methodology by employing small apertures and filters that avoided nebular lines, with subsequent transformation to the UBV system. However, the problem of the nebular continuum remained underappreciated. A planetary like NGC 7027 has a total continuum flux at $V$ equivalent to an eleventh magnitude star (Kaler 1976a), so it is in fact a crucial consideration for small objects. The next logical step, therefore, is to calculate it on the basis of observed nebular parameters, with procedures developed by Webster (1969) and, more elaborately, by Kaler (1976b, 1978a) and Martin (1981), who used the detailed theoretical results of Brown and Mathews (1970). This technique culminated in extensive studies by Shaw and Kaler $(1985,1988)$. They extracted the stellar flux from the whole by using measured or adopted electron temperatures and densities and helium ion abundances, and attached realistic errors to these quantities and propagated them through the calcula-tions. Their work showed the Shao and Liller data to be reliable for nebulae larger than about 40 arc seconds in diameter; smaller objects again yielded under-estimates.

However, this technique has its limitations too. As the stars get fainter relative to the nebulae in which they are embedded the magnitudes become very susceptible to errors in the input parameters, and become unreliable. The problem is especially severe for small high density nebulae for which the hydrogen $2 \mathrm{p} / 2 \mathrm{~s}$ population ratio, 
critical to the calculation of the two quantum continuum, is essentially unknown. Eventually, the stars become lost in the continuum flux. Comparison with other magnitude derivations suggests that the Shaw and Kaler $(1985,1988)$ results are reasonably reliable to roughly $\mathrm{V}=14.3$ and 15 respectively.

A natural extension of this technique would employ spectra rather than filters. Ideally, the stellar spectrum and the appropriate nebular diagnostics for the subtraction of the nebular continuum would be observed simultaneously. Méndez, Kudritzki, and Simon (1985), for example, converted their spectra into B magnitudes, but without such correction since it was deemed to be small. As a variation on this theme, we can use IUE spectra, which have the advantage that because the stellar flux rises so steeply into the UV, the contrast between the star and the nebular continuum is notably enhanced (Heap 1983). We can either use the UV fluxes directly in our analyses or convert them to visual magnitudes via an assumption of flux distribution and a measurement of interstellar extinction. The method, however, is quite sensitive to errors in extinction, as we must calculate the nebular continuum from the $\mathrm{H} \beta$ flux, or extrapolate the stellar flux over an equally long wavelength baseline to the visual. In addition, if the nebula is larger than the IUE (or HST!) aperture we do not even know the convolved $\mathrm{H} \beta$ flux, and we may well be uncertain as to our assumed stellar model. The UV has been extensively employed by Kaler and Feibelman (1985) and by Heap and Augensen (1987). The former authors studied only large nebulae so that no correction for the nebula was needed, and they converted their UV fluxes into $V$ magnitudes. The method is especially useful for unresolved nebulae with faint stars, and for planetaries whose nuclei are confused with nearby visual companions (e.g. K1-14); it is the only method that can be used for close binaries such as LoTr 5 (Feibelman and Kaler 1983).

These magnitudes are most commonly employed to calculate Zanstra temperatures and luminosities. The limitations on the methods discussed above conspire to produce a limit on the detectability of stars in compact nebulae, which in turn places a lower limit of roughly $125,000 \mathrm{~K}$ on the temperatures (Kaler 1986, Shaw and Kaler 1988), which is below the turnaround point on the $\log \mathrm{L}-\log \mathrm{T}$ plane (where the nuclei begin their descents to the white dwarf zone) for cores of $0.6 \mathrm{M}_{\odot}$ and up (Paczynski 1971; Schönberner 1979, 1983). The highest mass cores, however, of the order of $1.4 \mathrm{M}_{\odot}$, are predicted to reach up to $10^{6} \mathrm{~K}$. In order to study the hotter variety of stars we must apply sophisticated imaging techniques, in hopes of detecting a faint point source against the continuum background. Reay et al. (1984) so determined the magnitudes of 8 stars by examining the nebulae in narrow line-free wavelength bands, and Walton et al. (1986) present results on 21. This type of work culminated in the detection of the elusive nucleus of NGC 2440 by Atherton, Reay, and Pottasch (1986), at a visual magnitude of 18.9 and a record temperature of $350,000 \mathrm{~K}$. 
This method too is not without its limitations and problems. The above authors showed it to be very sensitive to seeing. And Heap (1987), who detected the NGC 2440 nucleus at the extreme short-wave capability of the IUE, finds it to be a magnitude brighter, and consequently about $150,000 \mathrm{~K}$ cooler. Obviously the imaging procedure is susceptible to inhomogeneities in a nebula that could affect the appearance of the assumed stellar source. To avoid ambiguities we would have to map the object in the continuum and in the diagnostic lines, then compute a map of the true nebular continuum and subtract it from that observed to isolate the pure star: a process not yet attempted.

In summary, the magnitudes of the planetary nuclei are distinct1y improving, but are still subject to serious uncertainties at the faint end, above about 15 th magnitude. We need to approach the problem with the variety of techniques outlined above, adapted to the kind of nebula being observed. There are extremes for which the choice is clear. Faint stars in extended bright objects can be detected only by careful imaging. For compact, or distant unresolved nebulae, we must resort to continuum subtraction by calculation. Both methods are aided by working in the UV, which is a necessity for a nucleus unresolved from a binary companion.

\section{SPECTRA}

The planetary nuclei occupy an enormous portion - roughly one quarter - of the extended $\log$ L-log T plane (e.g. see Pottasch 1984 Fig. IX-2), and we see a concomitant large variety of spectral morphologies that range from pure emission through emissionabsorption mixtures and near-continuous to pure strong absorption. Organization of the spectra is discussed in detail by Aller (1968, 1976, 1977), who classifies a large number of stars and presents many illustrative examples, by Smith and Aller (1969), and by Lutz (1978). We currently recognize Wolf-Rayet type spectra, 0 VI emission, of, WR-Of, continuous (which do not really exist if we look closely enough), and absorption- 0 .

The appearance of the spectrum depends upon temperature, luminosity, and chemical composition, or more fundamentally, upon core mass and state of evolution (Heap 1982). The nuclei that are on the descending portions of their evolutionary tracks lack winds because of their lowered luminosities (Heap 1982, Kaler and Feibelman 1985) and therefore have absorption spectra. Méndez, Kudritzki, and Simon (1985) and Méndez et al. (1987, hereafter MKHHG), demonstrate that some show large composition anomalies that presage those found among the white dwarfs.

On the horizontal evolutionary tracks we find more variety, as winds can develop toward higher luminosity. MKHHG for example demonstrate that for $\log \mathrm{T}>4.45$, He II $\lambda 4686$ passes from absorption 
to emission, producing an of type star, at a core mass of about 0.7 , or $\log \mathrm{L} \approx 4.1$. Stars with powerful carbon emissions are arrayed from the lowest detectable temperatures (M4-18, below 25,000 K: Goodrich and Dahari 1985), where C II and C III dominate, to over $10^{5}$ $\mathrm{K}$ (Smith and Aller 1969, Kaler and Shaw 1984) where C IV reigns and even C V is detected (Méndez and Niemela 1982).

These WC stars also exhibit a strong mixture of oxygen lines in their spectra, as well as those of silicon. 0 II is important at the cool limit, and in the $30,000 \mathrm{~K}$ range we find powerful 0 III and even the beginning of $0 \mathrm{~V}$ (Aller 1968). Oxygen really makes its presence felt above $80,000 \mathrm{~K}$ (Kaler and Shaw 1984) where O VI develops, creating the well-known $O$ VI stars, classified by Méndez and Niemela (1982) as WC2-WC4. At the extreme these latter authors even identify strong $O$ VII. The principal $O$ VI feature is the $3 s^{2} \mathrm{~S}-3 \mathrm{p}^{2} \mathrm{P}$ doublet at $\lambda 3811-\lambda 3834 \AA$. Wind speed is a strong function of effective temperature (Kaler, Mo, and Pottasch 1985), and at these high values these lines are usually blended into one with an effective wavelength of $3820 \AA$.

The critical luminosities needed for the development of the WC and $O$ VI phenomena are unknown. Although we are able to derive Zanstra temperatures for them, the distances are too insecure to enable the absolute bolometric magnitudes to be found. The constantmass (Shklovsky) distance method is not a fine enough discriminator to start with, the difficulty compounded by the optically thick natures of the nebulae with cool WC nuclei. And the appealing method developed in MKHHG does not work as these stars have no analyzable photospheric absorptions. Consequently we are ignorant about even fundamental matters concerning these remarkable windy stars.

Real progress will require considerable quantitative measurement of emission line fluxes with broad wavelength coverage. A strong start in this direction has been made by Aller (1977) and more recently by Aller and Keyes (1985), who treat a wide variety of objects. Extreme low and high excitation stars have been so examined by Goodrich and Dahari (1985) and Kaler and Shaw (1984) respectively. The winds that create these bizarre spectra are apparently quite significant in the evolution of planetary nuclei (Iben 1984), and it is important that we understand them better than we now do.

\section{TEMPERATURES}

The real controversies within the broad subject of stellar properties are reserved for temperatures. There are a variety of ways of approaching the problem and of deriving this critical parameter that are well-described in the literature: the classic Zanstra method, in which we compare the nebular recombination flux with the stellar magnitude (e.g. Harman and Seaton 1964); the energybalance (Stoy) method, which avoids the star by comparing nebular 
forbidden and recombination line fluxes (Kaler 1976c, Preite-Martinez and Pottasch 1983); analysis or modeling of the nebular ionic distribution (Natta, Pottasch, and Preite-Martinez 1980; Harrington and Feibelman 1983); UV energy distribution (Pottasch et al. 1978, Lutz and Carnochan 1979; Harrington et al. 1982; Clegg and Seaton 1983; Kaler and Feibelman 1985; Grewing and Bianchi 1987); diameter correlations developed from the latter (Amnuel et al. 1985); and the modeling of stellar absorption lines (Mendez, Kudritzki, and Simon 1985; MKHHG). Extensive lists of temperatures are given in several of the references of this section, as well as by Pottasch (1984) and Khromov (1985).

The methods do not yield good agreement with one another, carrying on an argument that has existed for nearly 50 years. The fundamental problem is that blackbody Zanstra temperatures based on He II, which derive from the stellar spectrum shortward of $\lambda 228 \AA$, are frequently higher (and often much higher) than those based on $H$, which use the integrated spectrum shortward of $\lambda 912 \AA$. Zanstra (1961) believed this discrepancy to be caused by deviations from a blackbody, i.e. a UV excess shortward of $\lambda 228$; Minkowski (1942) and Wurm (1951) thought it due to optical depth, wherein the true effective temperature should be set equal to $T_{z}$ (He II), the $H$ value being a lower limit caused by the escape of Lyman continuum radiation. The latter view has generally prevailed in the construction of evolutionary diagrams (Seaton 1966, Kaler 1983). However, it is clear that model atmospheres (e.g. Hummer and Mihalas 1970; Henry and Shipman 1986, hereafter HS; see Harrington and Feibelman 1983, and Pottasch 1984) really do show severe departures from the blackbody, seriously compromising many extant studies.

MKHGG's recent derivation of stellar properties by line-profile fitting provides an independent way of checking the blackbody Zanstra $\left(T_{z}\right)$ and Stoy $\left(T_{s}\right)$ temperatures. The comparison, in Figure 1 , shows $T_{z}$ and $T_{s}$ plotted against those found from the line fits. The $T_{z}$ above $60,000 \mathrm{~K}$ (open symbols) are all derived from He II according to common practice. The classic Zanstra discrepancy shows clearly in that the $\mathrm{T}_{2}$ (He II) are systematically too high, consistent with HS's flux distributions for low He abundances, which are indeed found for these stars by MKHHG. In fact the average amount of the discrepancy is $19,000 \mathrm{~K}$, nicely within the range of that anticipated by HS.

The $T_{z}(H)$ for these hot stars (filled symbols) all fall below $\mathrm{T}$ (MKHHG), which can still be explained by optical depth effects; these nebulae either exhibit little in the way of low excitation ions, or they possess outer shells that demonstrate the leakage of ionizing radiation. General support for the optical depth explanation comes from the correlation between the $T_{z}(H e I I) / T_{z}(H)$ ratios and the strengths of low excitation ions, which disappear when the ratios approaches high values (Kaler 1983). A particular argument against the lower MKHHG temperatures is NGC 7293 (the top point in Fig. 1), for which the $\mathrm{H}$ and He II Zanstra values agree. 


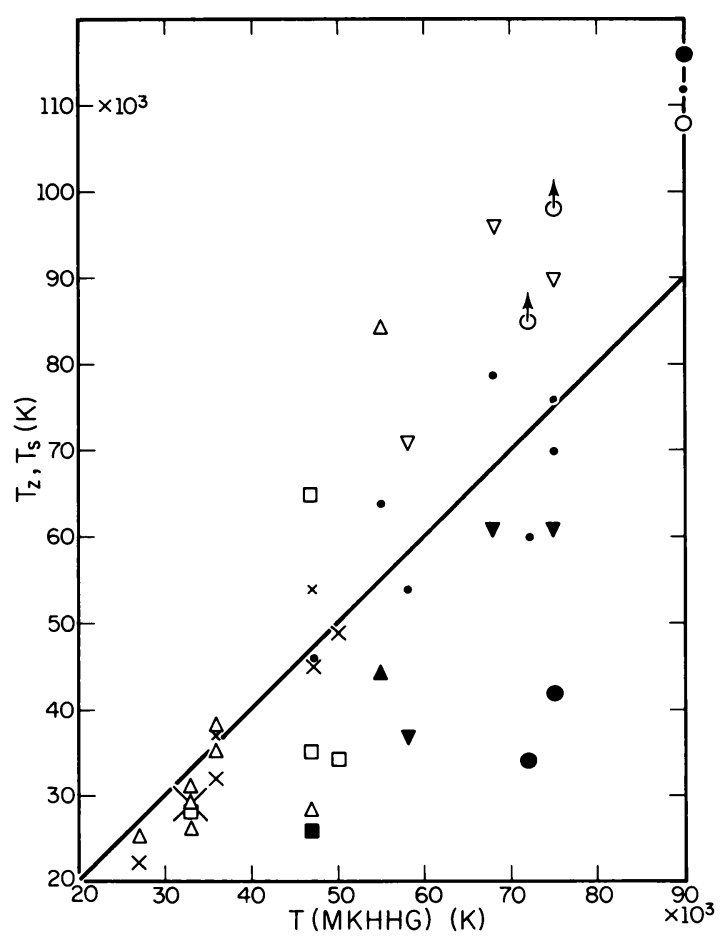

Fig. 1. $T_{2}$ and $T_{s}$ plotted against $\mathrm{T}$ (MKHHG). Above $\mathrm{T}_{2}$ $=60,000 \mathrm{~K}$ the open symbols represent He II temperatures; below it they indicate values derived from $\mathrm{H}$ for nebulae that do not exhibit He II. The filled symbols denote $T_{z}(H)$ for stars for which there are $\mathrm{T}_{\mathrm{z}}$ (He II) values. The small solid dots are the means of $T_{z}$ (He II) and $\mathrm{T}_{2}(\mathrm{H})$. Sources are $\odot$ : Kaler (1983); $\square, \Delta$ : Shaw and Kaler $(1985,1988) ; \nabla$ : this paper (NGC 1535, 3242, 7009 with corrected photographic magnitudes). The $\mathrm{X}$ symbols represent Stoy temperatures: small: estimated from $\lambda 5007$ and Kaler (1978b); middle size: computed values from Kaler (1976c,1978b); large: four points falling together.

Curiously, the average of $T_{z}$ (He II) and $T_{z}(H)$ (small dots) fits (probably fortuitously) with $\mathrm{T}$ (MKHHG) quite well. There may be no single answer to the Zanstra discrepancy: it may be caused by some combination of both effects, their relative importance depending upon such parameters as nebular size, and stellar temperature, luminosity, and composition.

Below $60,000 \mathrm{~K}$, where we must use $\mathrm{T}_{z}(\mathrm{H}$ ) (which should now be correct since these nebulae ought to be optically thick), the Zanstra temperatures still fall systematically below the MKHHG values, a result not predicted by HS. Systematic magnitude errors (Section I) could play a role. The stoy temperatures, however, actually agree rather well with MKHHG's results (although still a bit below them), so that we might be tempted to think that we can actually derive realistic values for these cooler stars.

A problem with the line profile temperatures arises from Kaler and Feibelman's (1985) finding that planetary nuclei can have Rayleigh-Jeans (infinite temperature) energy distributions in the accessible UV, far steeper than implied by the Zanstra temperatures. This problem has yet to be addressed: until the models used for stellar analysis can reproduce this odd characteristic (or the IUE 
observations are found to be flawed), we must be cautious about using the results derived from them.

The Kaler and Feibelman (1985) results have a more significant bearing on temperatures derived directly from UV energy distributions. It is hard to have confidence in any of the results so found when some of them are so obviously wrong. In order to interpret the slopes of the stellar continua that are flatter than Rayleigh-Jeans in terms of temperature, we must first be able to understand how the anomalously steep slopes can arise. Thus the 90,000 $\mathrm{K}$ temperature found for NGC 40 (whose Stoy temperature from Kaler 1976 is 32,000 K) by Grewing and Bianchi (1987) is probably too high, and like most of the values derived by Kaler and Feibelman (1985) should at least for now be considered an upper limit. The use of the continuum for cool WC stars is also compromised by the difficulty in locating it because of all the line features (Heap 1983; Kaler et al. 1988).

Finally, let us tie these three sections together by considering a temperature calibration of spectral class much as is traditionally done for the main sequence. Most of the planetary spectral classes (Of, WR-Of) are insufficiently described and subdivided, but the WC system given to us by Méndez and Niemela (1983) provides a good

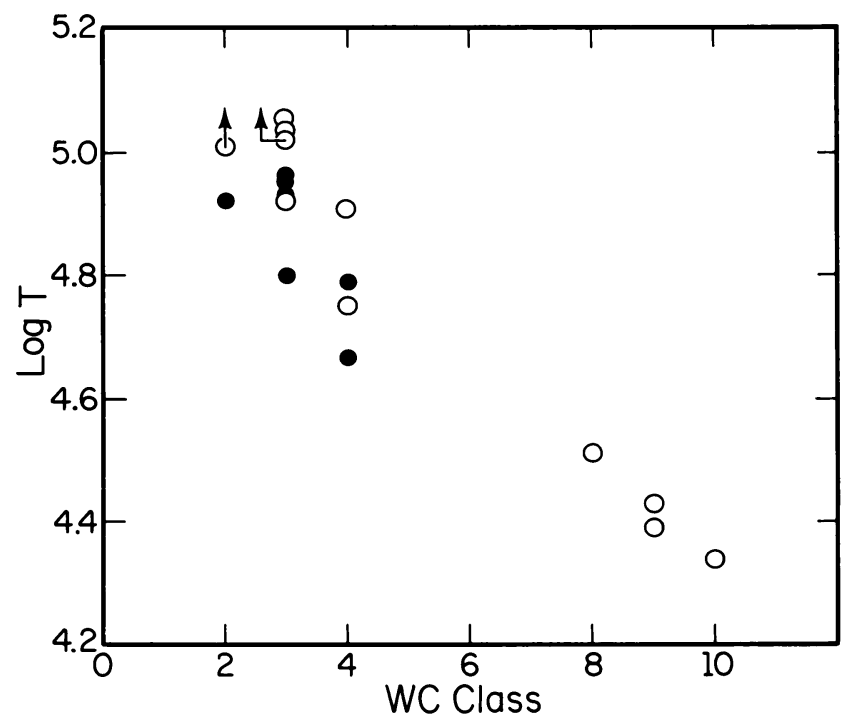

Fig. 2. Temperature calibration of the WC classification of Méndez and Niemela (1983). For early types (WC2-4) the open symbols represent $T_{z}$ (He II) from Kaler and Shaw (1984) and Shaw and Kaler (1988), and the closed symbols represent equivalent MKHHG temperatures, $\mathrm{T}_{z}$ (He II) lowered by $19,000 \mathrm{~K}$. Log $\mathrm{T}$ for NGC 5315 is an average of these with $\mathrm{T}_{\mathbf{s}}$ derived from $\lambda 5007$. For the later types (WC8-10) $\mathrm{T}_{\mathbf{s}}$ is used for NGC 40 and BD+30 (Kaler 1976c, 1978b) and M4-18 (Goodrich and Dahari 1985), and $T_{z}(H)$ for He2-99 from Shaw and Kaler (1988). 
opportunity. These are plotted in Figure 2 with $\mathrm{T}_{z}$ (He II) and the equivalent $\mathrm{T}$ (MKHHG) for the hot stars (WC2-4) and, with one exception, $T_{s}$ for the cool ones. The result is quite similar to that previously obtained by Méndez et al. (1986). We see a nice linear relationship, which means that as the temperatures improve and we are able to resolve the discrepancies among the methods, we will be able to infer $\mathrm{T}_{\text {eff }}$ directly from spectral class. The next step is to subdivide the other kinds of stars properly. One disconcerting aspect of this figure is the curious gap between WC4 and WC8, which implies that the hot WC class may not be directly related to the cool one. That does not compromise their calibration, however.

In summary, the past few years have seen some significant advances that include improved magnitudes and the refinement of temperature methods. There remain important unsolved questions such as the origin of the Zanstra discrepancy and the relative effects of optical depth, deviation of stellar energy distributions from blackbodies, and the effect of model-errors on the derived temperatures. In all, however, progress has been impressive.

\section{REFERENCES}

Abe11, G. 0. 1966, Ap. J., 144, 259.

Acker, A., Gleizes, F., Chopinet, M., Marcont, J., Ochsenbein, F., Roques, J. M. 1982, Catalogue of the Central Stars of True and Possible Planetary Nebulae, Pub1. Spec. du CDS, No. 3.

Aller, L. H. 1968, in Planetary Nebulae. IAU Symp. No. 34, ed. D. E. Osterbrock and C. R. O'Del1 (Dordrecht: Reidel), p. 339. 1976, Mém. Soc. Roy. Sci. Liège, Ser. 6, 9, 271. 1977, J. Roy. Astron. Soc. Canada, 71, 67.

Aller, L. H., and Keyes, C. D. 1985, Pub.A.S.P., 97, 1142.

Amnue1, P. R., Guseinov, 0. H., Novruzova, H. I., and Rustamov, Yu. S. 1985, Ap. Sp. Sci., 113, 59.

Atherton, P. D., Reay, N. K., and Pottasch, S. R. 1986, Nature, $320,423$.

Brown, R. L., and Mathews, W. G. 1970, Ap. J., 160, 939.

Clegg, R. E. S., and Seaton, M. J. 1983, in Planetary Nebulae. IAU Symp. No.103, ed. D. R. Flower (Dordrecht: Reide1), p. 536. Curtis, H. D. 1918, Lick Obs. Publ., 13, 57.

Feibelman, W. A., and Kaler, J. B. 1983, Ap. J., 269, 592.

Goodrich, R. W., and Dahari, 0. 1985, Ap. J., 289, 342.

Grewing, M., and Bianchi, L. 1987, Astr. Ap., in press.

Harrington, J. P., and Feibelman, W. A. 1983, Ap. J., 265, 258.

Harrington, J. P., Seaton, M. J., Adams, S., and Lutz, J. H. 1982, M.N.R.A.S., $199,517$.

Harman, R. J., and Seaton, M. J. 1964, Ap. J., 140, 824.

Heap, S. R. 1982, in Wolf-Rayet Stars: Observations, Physics, and Evolution. IAU Symp. No. 99, ed. C. de Loore and A. Willis (Dordrecht: Reide1), p. 423. 
. 1983, in Planetary Nebulae. IAU Symp. No. 103, ed. D. R.

Flower (Dordrecht: Reide1), p. 375.

- 1987, Nature, 326, 571.

Heap, S. R., and Augensen, H. J. 1987, Ap. J., 313, 268.

Henry, R. B. C., and Shipman, H. L. 1986, Ap. J., 311, 774 (HS).

Hummer, D. G., and Mihalas, D. 1970, in Surface Fluxes for Mode1 Atmospheres for the Central Stars of Planetary Nebulae (JILA Rpt. No. 101).

Iben, I. Jr. 1984, Ap. J., 377, 233.

Kaler, J. B. 1976a, Ap. Lett., 17, 163.

. 1976b, Ap. J., 210, 113 .

. $1976 \mathrm{c}, \mathrm{Ap.J.}, 210,843$.

1978a, Ap. J., 226, 947.

1978 b, Ap. J., 220, 887.

1983, Ap. J., 271, 188.

1986, Nature, $320,394$.

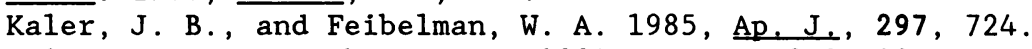

Kaler, J. B., and Shaw, R. A. 1984, Ap. J., 278, 195.

Kaler, J. B., Shaw, R. A., Feibelman, W. A., and Lutz, J. H. 1988, in preparation.

Kaler, J. B., Mo, J.-E., and Pottasch, S. R. 1985, Ap. J., 288, 305 .

Khromov, G. S. 1985, Planetary Nebulae.

Kostjakova, E. B., Savel'eva, M. V., Dokuchaeva, O. D., and Noskova, R. I. 1968, in Planetary Nebulae. IAU Symp. No. 38, ed. D. E. Osterbrock and C. R. O'Dell (Dordrecht: Reide1), p. 317.

Liller, W. 1978, in Planetary Nebulae. IAU Symp. No. 76, ed. Y. Terzian (Dordrecht: Reide1), p. 35.

Liller, W., and Shao, C.-Y. 1968, in Planetary Nebulae. IAU Symp. No. 34 , ed. D. E. Osterbrock and C. R. O'Dell (Dordrecht: Reide1), p. 320 .

Lutz, J. H. 1978, in Planetary Nebulae. IAU Symp. No. 76, ed. Y. Terzian (Dordrecht: Reide1), p. 185.

Lutz, J. H., and Carnochan, D. J. 1979, M.N.R.A.S., 189, 701.

Martin, W. 1981, Astr. Ap., 98, 328.

Méndez, R. H., Kudritzki, R. P., Herrero, A., Husfeld, D., and Groth, H. G. 1987, Astr. Ap., in press (MKHHG).

Méndez, R. H., Miguel, C. H., Heber, U., and Kudritzki, R. P. 1986, IAU Colloquium No. 87. Hydrogen Deficient Stars and Related Objects, ed. K. Hunger, D. Schönberner, and $N$. Kameswara (Dordrecht: Reide1), p. 323.

Méndez, R. H., Kudritzki, R. P., and Simon, K. P. 1985, Astr. Ap., $142,289$.

Méndez, R. H., and Niemela, V. S. 1982, in Wolf-Rayet Stars: Observations, Physics, Evolution, IAU Symp, 99, ed. C. W. H. de Loore and A. J. Willis, (Dordrecht: Reide1), p. 457.

Minkowski, R. 1942, Ap. J., 95, 243.

Natta, A., Pottasch, S. R., and Preite-Martinez, A. 1980, Astr. Ap., 84, 284.

Paczynski, B. 1971, Acta. Astr., 21, 417. 
Perek, L., and Kohoutek, L. 1967, Catalogue of Galactic Planetary Nebulae, Czechoslovak Acad. of Sci.

Pottasch, S. R. 1984, Planetary Nebulae, Ap. Sp. Sci. Lib., 107, (Dordrecht: Reidel).

Pottasch, S. R., Wesselius, P. R., Wu, C.-C., Fieten, H., and van Duinen, R. J. 1978, Astr. Ap., 62, 95.

Preite-Martinez, A., and Pottasch, S. R. 1983, Astr. Ap., 126, 31.

Reay, N. K., Pottasch, S. R., Atherton, P. D., and Taylor, K. 1984, Astr. Ap., 137, 113.

Schönberner, D. 1979, Astr. Ap., 79, 108. - 1983, private communication.

Seaton, M. J. 1966, M.N.R.A.S., 132, 113.

Shao, C.-C., and Liller, W. 1972, private communication.

Shaw, R. A., and Kaler, J. B. 1985, Ap. J., 295, 537. . 1988, in preparation.

Smith, L. F., and Aller, L. H. 1969, Ap. J., 157, 1245.

Walton, N. A., Reay, N. K., Pottasch, S. R., and Atherton, P. D. 1986, New Insights in Astrophysics, Proc. Joint NASA/ESA/SRC Conf., ESA SP-263.

Webster, B. L. 1969, M.N.R.A.S., 143, 113.

Wurm, K. 1951, Die Planetarischen Nebel (Berlin: Akademie-Verlag).

Zanstra, H. 1961, in Gaseous Nebulae and the Interstellar Medium, Univ. Mich. Ann Arbor, unpublished text. 


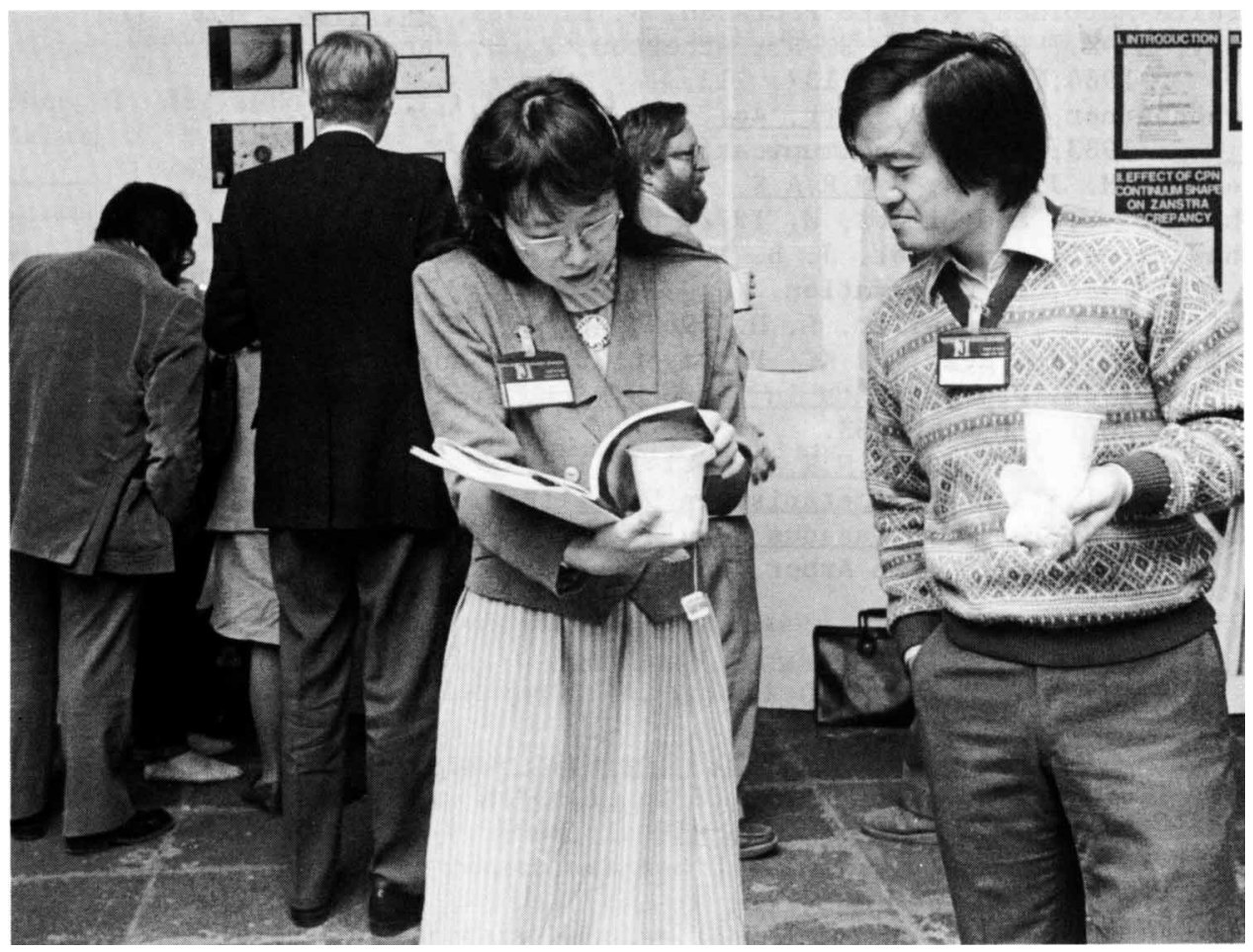

Marlko Kato and Izumi Hachisu. 